\title{
Basic science funding in Australia: lessons from the EU
}

\author{
Philip A. Stumbles', John W. Upham' and \\ DELIA J. NELSON²
}

'Telethon Institute for Child Health Research and Centre for Child Health Research, University of Western Australia, Australia. ${ }^{2}$ University Department of Medicine, QEII Medical Centre, University of Western Australia,Australia. (phils@ichr.uwa.edu.au)

We read with interest your recent Editorial on the $6^{\text {th }}$ Framework Programme for funding of research within the European Union (EU). Many of the key features (and problems) of this funding framework are mirrored in the current trends in basic science funding in Australia, particularly within our National Health and Medical Research Council

(NHMRC), the principal source of funding for basic medical research in Australia. In particular, we are concerned with the impact that this "big science" approach to funding will have on the ability of young investigators - including those that have yet to enter the NHMRC system as well as those that have begun to attract NHMRC funding but are not yet viewed as "independent" researchers - to establish independent career tracks. The ability to attract and retain emerging young investigators is a key foundation on which the future sustainability of such funding initiatives must be based.

The move towards increased funding of big collaborative science programs, as is the trend in the EU, with an emphasis on well established scientists with proven track records and histories of collaborative research, is now a stated objective of the Australian NHMRC and its New Program Grant (NPG) scheme.
To illustrate the importance ascribed to this scheme, NPGs are predicted to absorb the bulk of recent additional government funding allocated to the NHMRC and will continue to expand in the future, with no proposed expansion of the original Project Grant scheme that has been the traditional source of NHMRC funding.

In many cases, the problems identified in the EU concerning this approach, in particular peer review problems and stifling of innovation, are further exemplified in Australia by both our relatively small population base and lack of alternative nongovernment funding sources. Of more concern, however, is the potential for these systems to act towards preserving the status quo by channelling funds into research programs that are already well established and by reinforcing the "graying" of the research workforce.

The current direction of NHMRC funding poses a real dilemma for young investigators coming through the system. Tight restrictions on the number of NHMRC project grants held by chief investigators (only one outside their program) once they are part of a NPG severely hamper the ability of these senior scientists to collaborate and instigate new research initiatives with more junior researchers.

Consequently, emerging researchers are faced with a limited number of options. New investigators could attempt to preserve independence and research flexibility by staying outside the NPG scheme. However, these scientists now subject themselves to the difficulties of surviving within a highly competitive and underfunded NHMRC project grant scheme that has a current success rate of approximately $25 \%$. With such strong emphasis placed on previous track records, early success in this scheme often means being aligned with established researchers with good track records, many of whom will become associated with NPGs and thus have a limited ability to collaborate. Alternatively, they can join a large NPG. This will offer a greater degree of stability but this comes at a price: their ability to develop independent research paths (which the current system demands for them to succeed) is restricted, as is their ability to collaborate within the NHMRC scheme. Finally, they may look for alternative sources of funding, which in Australia often mean industrial or other sources that will not necessarily be to the benefit of basic science.

The solutions to this dilemma are likely to be complicated and require a greater level of consultation than has previously existed. Of course, at the outset there can be no substitute for increased levels of funding for basic science. However, we believe that it is not simply a matter of injecting more money into the system, but rather ensuring that existing or extra funding is carefully targeted in the right direction. Greater levels of cooperation between government, university departments and research institutes must be encouraged in order to support the careers of promising young research-only scientists. Stronger emphasis must be placed on "mentoring" young scientists through the system from an early point in their career, both by senior scientists and the bureaucrats that control science funding. Among other things, this will mean greater provision of skills required to survive within these systems, actively promoting and supporting collaboration between young investigators, both nationally and internationally, and greater provision of funding that is not so tightly aligned to established track records. Ultimately, the long-term success of the Australian, European and other similar national schemes depends on getting this right. 